\title{
A non-small cell lung cancer (NSCLC) patient harboring a rare epidermal growth factor receptor (EGFR) L858R/V843I mutation complex benefited from osimertinib: a case report
}

\author{
Yonghong Zhou ${ }^{1}$, Xiaofang $\mathrm{Qi}^{2}$, Yan Wang ${ }^{2}$, Hui Dong ${ }^{1}$, Li Zhuang ${ }^{1}$ \\ ${ }^{1}$ Department of Thoracic Surgery, Yunnan Cancer Hospital, Kunming, China; ${ }^{2}$ OrigiMed, Shanghai, China \\ Correspondence to: Li Zhuang, MD. Department of Thoracic Surgery, Yunnan Cancer Hospital, Kunzhou Road, Kunming 650118, China. \\ Email: zl19925805515@163.com.
}

\begin{abstract}
Tyrosine kinase inhibitors (TKIs) have greatly improved the survival of non-small cell lung cancer (NSCLC) patients with epidermal growth factor receptor (EGFR)-TKI sensitive mutations such as L858R and exon 19 deletions. The third-generation EGFR-TKI osimertinib, an irreversible TKI, is approved as a therapy for advanced NSCLC with EGFR sensitive mutations. Recently, osimertinib showed antitumor activity against NSCLC in patients harboring an uncommon mutation such as an exon 20 insertion. Herein, we present a patient diagnosed with stage IV NSCLC with an EGFR L858R/V843I mutation complex who benefited remarkably from osimertinib therapy. The patient was a 41-year-old Chinese female who complained of backache in October 2018. Computed tomography (CT) and magnetic resonance imaging (MRI) scans showed a mass in the right lung and brain metastasis. A whole-body bone scan revealed bone metastases. Targeted next-generation sequencing (NGS) of hydrothorax was performed and the coexistence of somatic L858RI and V843I mutations in EGFR exon 21 was discovered on November 13, 2018. Osimertinib therapy ( $80 \mathrm{mg}$ daily) was administered for 12 months which resulted in an initial partial response (PR). At that point, the right lower lung lesion enlarged, indicating disease progression. Thus, the patient began combination therapy with osimertinib (80 $\mathrm{mg}$ daily) and bevacizumab (500 mg daily), leading to disease stabilisation until June 2020. Of note, during treatment, the patient achieved sustained control of metastatic brain and bone lesions. To the best of our knowledge, we report here the first known case of an NSCLC patient with a somatic EGFR L858R/V843I mutation complex who responded well to osimertinib. Our findings provide theoretical guidance and expand the list of potential beneficiaries of EGFR-TKI therapy.
\end{abstract}

Keywords: Osimertinib; non-small cell lung cancer (NSCLC); tyrosine kinase inhibitor (TKI); epidermal growth factor receptor $(E G F R)$; case report

Submitted Aug 22, 2021. Accepted for publication Oct 20, 2021.

doi: 10.21037/apm-21-2653

View this article at: https://dx.doi.org/10.21037/apm-21-2653

\section{Introduction}

Epidermal growth factor receptor $(E G F R)$ is the most common driver gene accounting for $50 \%$ of Chinese nonsmall cell lung cancer (NSCLC) cases (1). Two types of mutations, exon 21 L858R substitutions and exon 19 deletions, are known as common mutations or classic mutations due to their large proportion and sensitivity to EGFR tyrosine kinase inhibitors (TKIs) (2). So far, the
FDA has approved 11 EGFR-TKIs for the treatment of NSCLC with EGFR common mutations. However, another $10-20 \%$ of uncommon mutations of EGFR including exon 18 mutations and exon 20 insertions, and other rare mutations that may benefit from EGFR-TKIs still remain uncertain. Also, complex mutations occur in $3.74 \%$ of Chinese NSCLC (3). The introduction of an additional mutation may change the molecular conformation of the 
EGFR tyrosine kinase domain, leading to change TKI affinity that subsequently affects the clinical outcome (4). Currently, there is no consensus opinion regarding the treatment of patients with EGFR uncommon mutations. The clinical case reports of rare mutations are of great reference value.

Osimertinib, a third-generation EGFR-TKI targeting irreversible classic mutations and the T790M mutation of $E G F R$, showed superior efficacy to first- or secondgeneration EGFR-TKIs in an updated FLAURA study, and is approved as a therapy for advanced NSCLC with EGFR sensitive mutations (5-7). A pre-clinical study showed a lower IC50 value and more sensitivity of secondor third-generation EGFR-TKIs compared with firstgeneration EGFR-TKIs for EGFR uncommon mutations (8). Clinical studies also found that second-generation showed superiority over the gefitinib or erlotinib in median ORR (62.5\% vs. 50\%) and PFS (11.0 vs. 3.6 months) (9). Recently, a phase II clinical trial enrolled 37 NSCLC patients harboring uncommon EGFR mutations, including G719X, L861Q, S768I, and others, who received osimertinib $(80 \mathrm{mg})$ as a first-line treatment. Osimertinib showed a $50 \%$ objective response rate and 8.2 months median progressionfree survival, which indicates favorable antitumor activity against NSCLC in patients harboring an uncommon mutation (10). It appears that afatinib and osimertinib are better options for these patients, however, a recently study showed that patients with complex EGFR mutations and a secondary T790M mutation have a lower ORR (27\%), shorter PFS (2.9 months) and OS (17.8 months) when subsequently treated with osimertinib (11). Considering this, first-line osimertinib maybe a choice for these patients.

The frequency of EGFR V843I substitution mutation is very low in lung cancer patients. We only found 3 NSCLC patients harboring the V843I mutation among 30,454 patients $(12,13)$. However, previous studies have demonstrated that the EGFR V843I mutation contributes to tumorigenesis and provides resistance to EGFR-TKIs via structural modifications of $E G F R$ comparable to those in the context of the EGFR T790M mutation $(14,15)$. Here, we present a patient diagnosed with stage IV NSCLC with an EGFR L858R/V843I mutation complex who benefited remarkably from osimertinib therapy. To the best of our knowledge, this is the first known case of an NSCLC patient with a somatic EGFR L858R/V843I mutation complex who responded well to osimertinib.

We present the following article in accordance with the CARE reporting checklist (available at https://apm. amegroups.com/article/view/10.21037/apm-21-2653/rc).

\section{Case presentation}

A 41-year-old non-smoking Chinese female was admitted to our hospital in October 2018 with complaints of backache. Computed tomography and magnetic resonance imaging scans showed a lesion $(5.0 \mathrm{~cm} \times 4.4 \mathrm{~cm})$ in the right lung and brain metastasis (Figure 1A). A whole-body bone scan revealed bone metastases (Figure $1 B$ ), multiple nodules in both lungs, slightly larger lymph nodes in the mediastinum, thickening of the right pleura, and moderate effusion in the right pleural cavity. We performed thoracic puncture and drainage, and $1,800 \mathrm{~mL}$ yellow pleural fluid was drawn out. Cytological examination detected adenocarcinoma cells in the pleural fluid. She was diagnosed with lung adenocarcinoma (T2N2M1, stage IV) based on the results of hematoxylin and eosin staining (Figure 2A). Mutation profiling of blood and pleural fluid using circulating tumor DNA targeted next-generation sequencing (NGS) for 450 cancer-related genes was performed. Coexistence of somatic L858RI and V843I mutations in EGFR exon 21 was detected in both blood and pleural fluid (Figure 2B).

Osimertinib therapy ( $80 \mathrm{mg}$ daily) combined with whole brain radiation therapy (WBRT) (30 Gy/3 times) for the whole brain and gross target volume (GTV) (40 Gy/10 times) for other metastasis lesions was initiated on November 15, 2018. The treatment induced a partial response $(\mathrm{PR}, 1.9 \mathrm{~cm} \times 0.9 \mathrm{~cm})$, which continued for 12 months. At that point, the right lower lung lesion enlarged $(3.4 \mathrm{~cm} \times 3.2 \mathrm{~cm})$, indicating disease progression. Thus, the patient began combination therapy with osimertinib $(80 \mathrm{mg}$ daily) and bevacizumab (500 $\mathrm{mg}$ daily), leading to disease stabilisation until June 2020. Of note, during treatment, the patient achieved sustained control of metastatic brain and bone lesions, and no adverse event was observed.

All procedures performed in this study were in accordance with the ethical standards of the institutional and/or national research committee(s) and with the Helsinki Declaration (as revised in 2013). Written informed consent was obtained from the patient for publication of this case report and accompanying images. A copy of the written consent is available for review by the editorial office of this journal.

\section{Discussion}

This is a very rare case of a patient with the L858R/ V843I compound somatic mutation who benefited from 


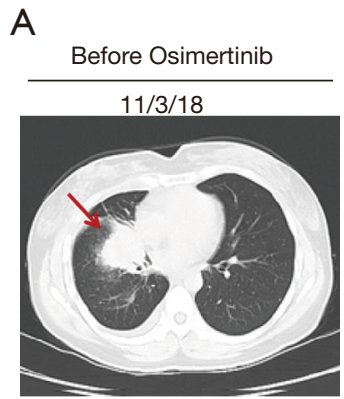

$5.0 \mathrm{~cm} \times 4.4 \mathrm{~cm}$

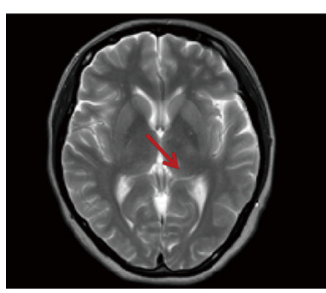

Left dorsal thalamus: $0.7 \mathrm{~cm}$ Right insular cortex: $0.4 \mathrm{~cm}$

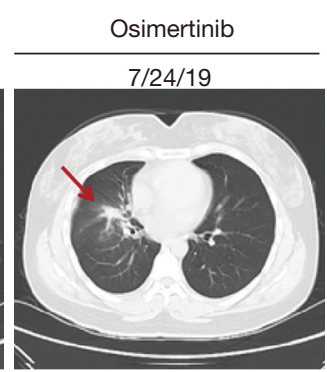

$1.9 \mathrm{~cm} \times 0.9 \mathrm{~cm}$

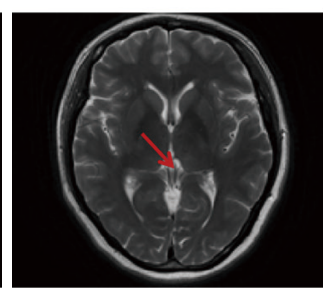

Left dorsal thalamus: $0.2 \mathrm{~cm}$ Right insular cortex: $0 \mathrm{~cm}$

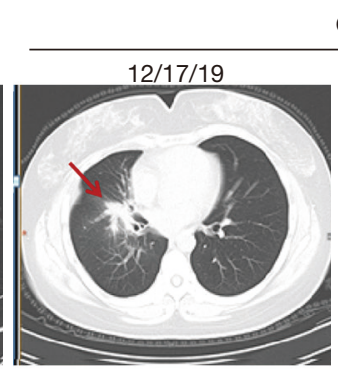

$3.4 \mathrm{~cm} \times 2.2 \mathrm{~cm}$

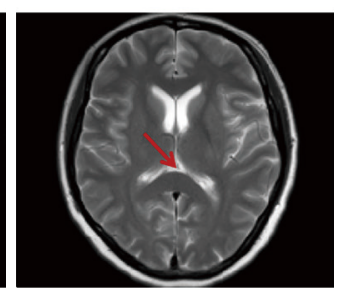

Left dorsal thalamus: $0.5 \mathrm{~cm}$ Right insular cortex: $0 \mathrm{~cm}$
Osimertinib + Bevacizumab

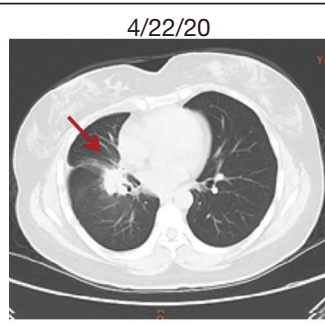

$2.5 \mathrm{~cm} \times 2.3 \mathrm{~cm}$

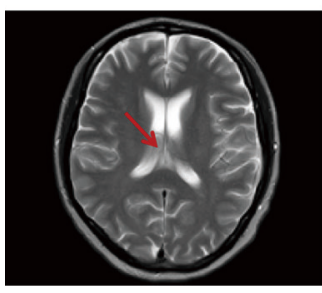

Left dorsal thalamus: $0.3 \mathrm{~cm}$ Right insular cortex: $0 \mathrm{~cm}$

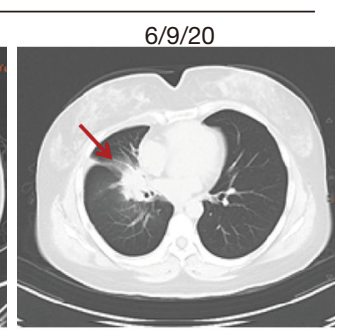

$3.5 \mathrm{~cm} \times 2.6 \mathrm{~cm}$

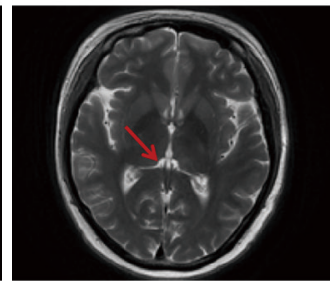

Left dorsal thalamus: $0.3 \mathrm{~cm}$ Right insular cortex: $0 \mathrm{~cm}$
B $11 / 3 / 18$
$7 / 25 / 19$

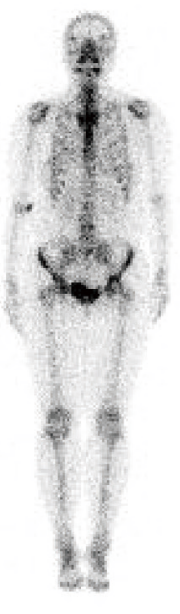

R ANT L

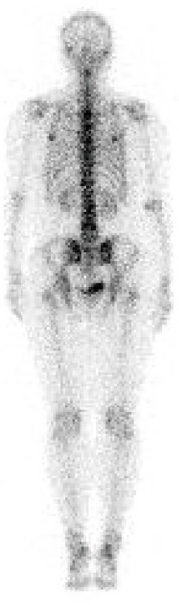

L POST R

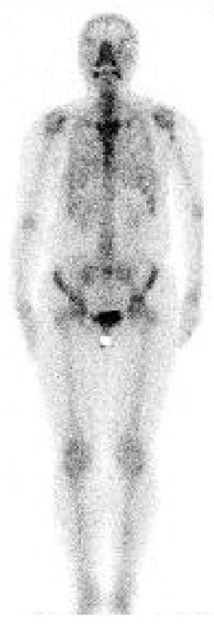

R ANT L
$12 / 18 / 19$

Figure 1 Disease timeline for lung adenocarcinoma and brain/bone metastases. (A) Magnetic resonance imaging, computed tomography, and scans of the right lung and brain, respectively, illustrating the clinical response to different treatments (red arrows point to lesions); (B) bone metastases revealed by whole-body bone scanning. ANT, anterior; L, left; POST, posterior; R, right.

osimertinib. The role of the V843I substitution in EGFR is poorly understood, probably because of its low frequency in lung cancer patients. Evidence on the effectiveness of EGFR-TKIs against advanced lung adenocarcinoma with the L858R/V843I compound mutation is rare, and only sporadic cases with germline V843I mutations have been reported. Of note, an initial report revealed that patients with the germline V843I mutation showed resistance to erlotinib (16). Pre-clinical studies using the KCL-PLA1 cell line which harbors a L858R/V843I compound mutation showed resistance to a second-generation EGFR-TKI (14). We found only one Chinese female NSCLC patient with an EGFR V843I germline mutation who received osimertinib and then achieved PR for 7 months (17). As previously 

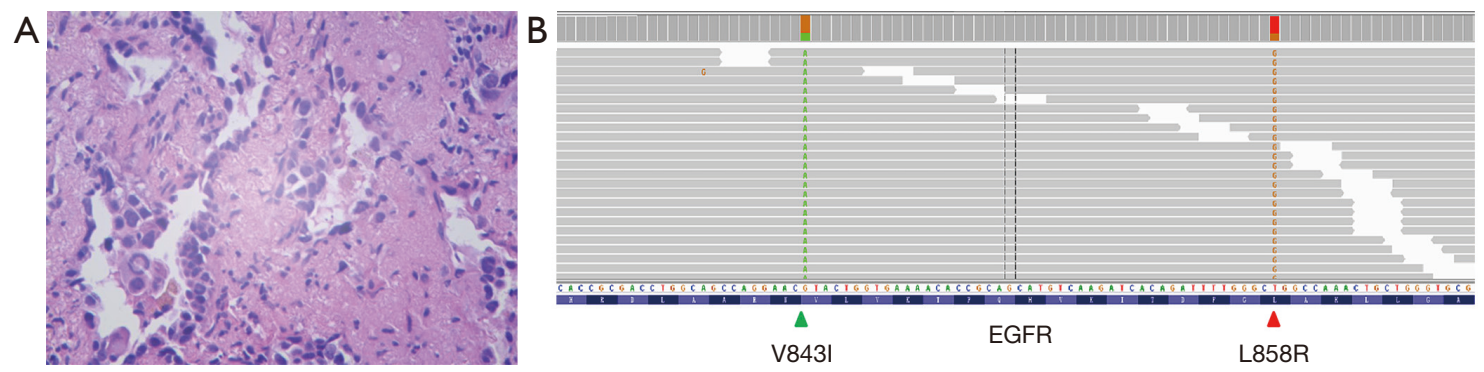

Figure 2 Lung adenocarcinoma in a 41-year-old Chinese female. (A) Hematoxylin and eosin staining (original magnification $\times 400)$ of lung tissue; (B) EGFR L858R and V843I double-point mutations were identified in tissues.

reported, TKI binding to EGFR would be sterically hindered by Arg841 in the double-mutant (V843I + L858R) EGFR resulting multiple TKI resistance (13). However, how did osimertinib works still remain unknown. The possible mechanism is that osimertinib could easily bind to the EGFR kinase domain irreversibly by targeting the cysteine-797 residue because of its mono-anilino-pyrimidine compound 3D structure, avoiding sterically hindering caused by L858R/V843I. Additional studies on osimertinib efficacy focused specifically on EGFR L858R/V843I mutation patients are needed. To the best of our knowledge, we report here the first known case of an NSCLC patient with a somatic EGFR L858R/V843I mutation complex who responded well to osimertinib.

Reports of rare EGFR mutations support the decision making process in the treatment of patients with NSCLC. It's a big challenge to compare different EGFR-TKI or other therapy in a prospective study due to highly heterogeneous and low frequencies of EGFR uncommon mutation. Second-generation or third-generation TKI may be considered as first-line treatment for most EGFR uncommon mutations based on several retrospective studies (7-9). Our findings provide theoretical guidance and expand the list of potential beneficiaries of EGFR-TKI therapy. Nevertheless, more studies are needed to deepen our understanding of EGFR mutations and response to TKIs.

\section{Acknowledgments}

Funding: This work was supported by the Wu Jieping Medical Fund (No. 320.6750.18533).

\section{Footnote}

Reporting Checklist: The authors have completed the CARE reporting checklist. Available at https://apm.amegroups. com/article/view/10.21037/apm-21-2653/rc

Conflicts of Interest: All authors have completed the ICMJE uniform disclosure form (available at https:// apm.amegroups.com/article/view/10.21037/apm-212653/coif). All authors reported this work was supported by the Wu Jieping Medical Fund under Grant number 320.6750.18533. XQ and YW report they received personal fees from OrigiMed. The authors have no other conflicts of interest to declare.

Ethical Statement: The authors are accountable for all aspects of the work in ensuring that questions related to the accuracy or integrity of any part of the work are appropriately investigated and resolved. All procedures performed in this study were in accordance with the ethical standards of the institutional and/or national research committee(s) and with the Helsinki Declaration (as revised in 2013). Written informed consent was obtained from the patient for publication of this case report and accompanying images. A copy of the written consent is available for review by the editorial office of this journal.

Open Access Statement: This is an Open Access article distributed in accordance with the Creative Commons Attribution-NonCommercial-NoDerivs 4.0 International License (CC BY-NC-ND 4.0), which permits the noncommercial replication and distribution of the article with the strict proviso that no changes or edits are made and the original work is properly cited (including links to both the formal publication through the relevant DOI and the license). See: https://creativecommons.org/licenses/by-nc-nd/4.0/.

\section{References}

1. Wang S, Ma P, Ma G, et al. Value of serum tumor 
markers for predicting EGFR mutations and positive ALK expression in 1089 Chinese non-small-cell lung cancer patients: A retrospective analysis. Eur J Cancer 2020;124:1-14.

2. Kim EY, Cho EN, Park HS, et al. Compound EGFR mutation is frequently detected with co-mutations of actionable genes and associated with poor clinical outcome in lung adenocarcinoma. Cancer Biol Ther 2016;17:237-45.

3. Fang W, Huang Y, Hong S, et al. EGFR exon 20 insertion mutations and response to osimertinib in non-small-cell lung cancer. BMC Cancer 2019;19:595.

4. Shih JY, Gow CH, Yang PC. EGFR mutation conferring primary resistance to gefitinib in non-small-cell lung cancer. N Engl J Med 2005;353:207-8.

5. Soria JC, Ohe Y, Vansteenkiste J, et al. Osimertinib in Untreated EGFR-Mutated Advanced Non-Small-Cell Lung Cancer. N Engl J Med 2018;378:113-25.

6. Ramalingam SS, Vansteenkiste J, Planchard D, et al. Overall Survival with Osimertinib in Untreated, EGFR-Mutated Advanced NSCLC. N Engl J Med 2020;382:41-50.

7. $\mathrm{Wu} \mathrm{YL}$, Tsuboi M, He J, et al. Osimertinib in Resected EGFR-Mutated Non-Small-Cell Lung Cancer. N Engl J Med 2020;383:1711-23.

8. Kobayashi Y, Mitsudomi T. Not all epidermal growth factor receptor mutations in lung cancer are created equal: Perspectives for individualized treatment strategy. Cancer Sci 2016;107:1179-86.

9. Shen YC, Tseng GC, Tu CY, et al. Comparing the effects of afatinib with gefitinib or Erlotinib in patients with advanced-stage lung adenocarcinoma harboring nonclassical epidermal growth factor receptor mutations. Lung Cancer 2017;110:56-62.

Cite this article as: Zhou Y, Qi X, Wang Y, Dong H, Zhuang L. A non-small cell lung cancer (NSCLC) patient harboring a rare epidermal growth factor receptor (EGFR) L858R/V843I mutation complex benefited from osimertinib: a case report. Ann Palliat Med 2022;11(3):1121-1125. doi: 10.21037/ apm-21-2653
10. Cho JH, Lim SH, An HJ, et al. Osimertinib for Patients With Non-Small-Cell Lung Cancer Harboring Uncommon EGFR Mutations: A Multicenter, OpenLabel, Phase II Trial (KCSG-LU15-09). J Clin Oncol 2020;38:488-95.

11. Lin YT, Tsai TH, Wu SG, et al. Complex EGFR mutations with secondary T790M mutation confer shorter osimertinib progression-free survival and overall survival in advanced non-small cell lung cancer. Lung Cancer 2020;145:1-9.

12. Faehling M, Schwenk B, Kramberg S, et al. Oncogenic driver mutations, treatment, and EGFR-TKI resistance in a Caucasian population with non-small cell lung cancer: survival in clinical practice. Oncotarget 2017;8:77897-914.

13. Shih JY, Gow CH, Yu CJ, et al. Epidermal growth factor receptor mutations in needle biopsy/aspiration samples predict response to gefitinib therapy and survival of patients with advanced nonsmall cell lung cancer. Int J Cancer 2006;118:963-9.

14. Matsushima S, Ohtsuka K, Ohnishi H, et al. V843I, a lung cancer predisposing EGFR mutation, is responsible for resistance to EGFR tyrosine kinase inhibitors. J Thorac Oncol 2014;9:1377-84.

15. Ohtsuka K, Ohnishi H, Fujiwara M, et al. Predisposition to Lung Adenocarcinoma in a Family Harboring the Germline EGFR V843I Mutation. JCO Precis Oncol 2019.

16. Demierre N, Zoete V, Michielin O, et al. A dramatic lung cancer course in a patient with a rare EGFR germline mutation exon 21 V843I: Is EGFR TKI resistance predictable? Lung Cancer 2013;80:81-4.

17. Song H, Chen Y, Yan Z, et al. Response to Osimertinib in a NSCLC Patient Harboring EGFR V843I Germ-Line Mutation. Lung Cancer 2020;150:247-8. 